\title{
Measuring women's experiences of maternity care: protocol for a systematic review of self-report survey instruments
}

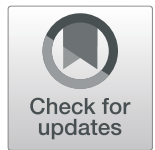

Claire Beecher $^{1^{*}}$ (D), Richard Greene ${ }^{2}$, Laura O'Dwyer ${ }^{3}$, Ethel Ryan ${ }^{4}$, Mark White $^{5}$, Michelle Beattie ${ }^{6}$ and Declan Devane $e^{1,7,8}$

\begin{abstract}
Background: The use of survey instruments to measure women's experiences of their maternity care is regarded internationally as an indicator of the quality of care received. To ensure the credibility of the data arising from these instruments, the methodological quality of development must be high. This paper reports the protocol for a systematic review of self-report instruments used to measure women's experiences of their maternity care.

Methods: Citation databases CINAHL, Ovid MEDLINE and EMBASE will be searched from 2002 to 2018 using keywords including women, experience, maternity care, questionnaires, surveys, and self-report. Citations will be screened by two reviewers, in two rounds, for inclusion as per predetermined inclusion and exclusion criteria. Data extraction forms will be populated with data, extracted from each study, to evaluate the methodological quality of each survey instrument and the criteria for good measurement properties using quality criteria. Data will also be extracted to categorise the items included in each survey instrument. A combination of a structured narrative synthesis and quantitate summaries in tabular format will allow for recommendations to be made on the use, adaptation and development of future survey instruments.
\end{abstract}

Discussion: The value of survey instruments that evaluate women's experiences of their maternity care, as a marker of quality care, has been recognised internationally with many countries employing the use of such instruments to inform policy and practice. The development of these instruments must be methodologically sound and the instrument itself fit for the purpose and context in which it is used. This protocol describes the methods that will be used to complete a systematic review that will serve as a guide for choosing the most appropriate existing instruments to use or adapt so that they are fit for purpose, in addition to informing the development of new instruments.

Systematic review registration: PROSPERO CRD42018105325

Keywords: Midwifery, Maternity, Measurement, Experiences of care, Quality care, Systematic review protocol, Instruments, Questionnaires, Surveys

\footnotetext{
* Correspondence: c.beecher1@nuigalway.ie

'School of Nursing and Midwifery, National University of Ireland, Galway,

Ireland

Full list of author information is available at the end of the article
}

(c) The Author(s). 2020 Open Access This article is distributed under the terms of the Creative Commons Attribution 4.0 International License (http://creativecommons.org/licenses/by/4.0/), which permits unrestricted use, distribution, and reproduction in any medium, provided you give appropriate credit to the original author(s) and the source, provide a link to the Creative Commons license, and indicate if changes were made. The Creative Commons Public Domain Dedication waiver (http://creativecommons.org/publicdomain/zero/1.0/) applies to the data made available in this article, unless otherwise stated. 


\section{Background}

The measurement of quality healthcare from the service users' perspective is a crucial element in the application of quality assurance and improvement processes $[1,2]$. Much of the quantitative measurement of the quality of care from the service user's perspective has focused on two aspects, i.e. satisfaction and experiences of care. The use of survey instruments to measure satisfaction with healthcare dates back to the 1950s [3]. However, the adequacy of satisfaction as a measure of quality has been questioned because it is indirectly related to the quality of care received [4, 5]. Satisfaction is also, generally, rated high by service users regardless of the quality of care that has been received, and has been attributed to a reluctance to criticise caregivers, as well as service users valuing what is known, or available, to them $[6,7]$. Given these limitations, the use of survey instruments that focus on the measurement of experiences of care, as an indicator of quality, has become more prominent. In contrast to satisfaction, experiences of care focus on accounts of the care received [8,9]. Survey instruments that measure experiences of care minimise the need for respondents to make evaluations on their care and focus on the reporting of what did or did not happen [9].

A reliance on the measurement of experiences of care to inform policy and practice is evident within maternity services [10-12]. There are, however, many challenges to measuring women's experiences of their maternity care. Maternity care is complex, encompassing numerous services at various time points, with a wide variety of professions and professionals along a temporal care continuum [13]. Furthermore, models of maternity care vary significantly between jurisdictions. The complex nature of maternity care coupled with the variances in services internationally has led to a proliferation of instruments that each seek to gather data on the quality of various aspects of these services from the perspective of women as service users [4].

Measurement of women's experience of maternity care requires robust instruments. Clinicians, managers, policymakers, and researchers must have access to the processes of development and measurement properties of these instruments when using or adapting existing instruments or developing new instruments to ensure that the resulting data, often used to direct policy and practice, is credible $[14,15]$. The aim of this review is to systematically review and critically appraise self-report survey instruments measuring women's experiences of their maternity care. The results of this review will serve as the basis of future research on the use, adaptation and development of self-report survey instruments internationally to measure women's experiences of their maternity care. For example, within the republic of Ireland specifically, these results will inform the development of the National Maternity Experience Survey (see https:// yourexperience.ie/maternity/about-the-survey/).

The objectives of the review are to

1. Identify self-report survey instruments that are available internationally to measure women's experiences of their maternity care;

2. Categorise items included within each survey instrument;

3. Evaluate the methodological quality of each survey instrument; and

4. Evaluate the criteria for good measurement properties using quality criteria.

\section{Methods and design \\ Design and registration}

The review protocol was submitted to the International Prospective Register of Systematic Reviews (PROSPERO) on 14 August 2018 (No. CRD42018105325). The protocol has been developed in accordance with the Preferred Reporting Items for Systematic Reviews and MetaAnalysis Protocols (PRISMA-P) statement [16] and the completed review will be reported in accordance with the PRISMA guidelines [17].

\section{Search strategy}

An extensive literature review will be performed and refined using the following citation databases: CINAHL, OVID Medline and Embase. Searching will be limited to literature published from 2002 onwards. This is based on a literature search by Messent [18] who found that up until this point, no published maternity survey instruments had been validated.

An example of a complete search to be carried out is included in Table 1. The search strategy was developed iteratively based on the authors experience (including experienced clinicians and systematic reviewers) and a review of strategies in related reviews. The strategy was then tested in three databases and revised to achieve the best balance between sensitivity and specificity of retrieved citations. The search strategy included in Table 1 has been formulated for use in the CINAHL database and will be adapted to respective databases.

$\mathrm{MH}$ represents CINAHL headings. N5 represents the number of words that could appear between keywords/ phrase

\section{Data screening}

Once all database searches have been completed, citations will be exported to the reference manager software Endnote X7 and duplicates removed. Remaining citations will then be exported to Covidence [19] and screened by two reviewers for inclusion using predetermined inclusion and exclusion criteria, set out in the following section. The 
Table 1 Search strategy for CINAHL database

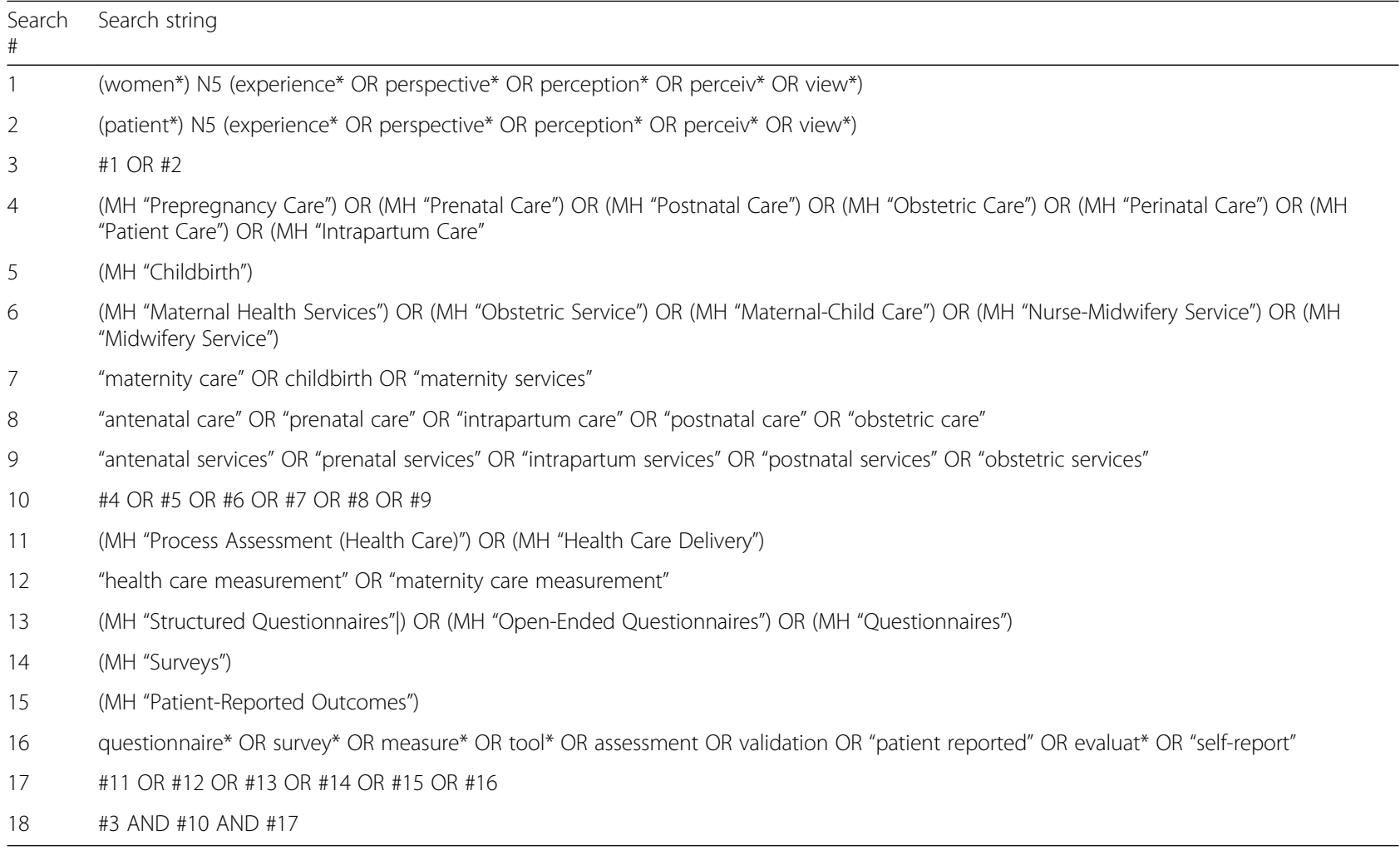

first round of screening will assess eligibility based on title and abstract. Round two of screening will assess the eligibility of all remining studies based on a full-text review. All abstracts and full texts will be sourced as necessary. Disagreements will be resolved through consensus with a third reviewer. Reasons for exclusion decisions at full-text (round 2) will be documented. References of included papers will be analysed for additional literature on the theoretical, empirical and psychometric development of instruments not identified in the original searches.

\section{Inclusion and exclusion criteria Inclusion criteria}

1. Literature that describes the theoretical or empirical development, or tests the psychometrics, of selfreport instruments that measure women's experiences of their maternity care;

2. Literature that focuses on self-report instruments that measure women's experiences of their maternity care from the perspective of women, rather than staff, families or others;

3. English language;

4. Primary research;

5. Literature that focuses on women's perceptions or views on their care are to be included as often these terms are used interchangeably with 'experiences';
6. Literature that focuses on the measurement of women's experiences of their entire maternity care process (from conception up to ten days postpartum), rather than one temporal aspect of care specifically, e.g. antenatal care; and

7. Literature that focuses on the measurement of experiences of maternity care as received by women in general, rather than a focus on participants by specific demographics, e.g. teenage pregnancy.

\section{Exclusion criteria}

1. Case reports and series, systematic reviews or metaanalysis;

2. Literature that focuses on indirect evidence of measurement properties of an instrument; for example, if an instrument is being used within a randomised controlled trial or alternative study, or if the instrument is being used as part of the validation process of an alternative instrument. This exclusion criterion is based on the recommendation of Terwee et al. [20] who suggest that not only is the process of sourcing this literature difficult but it is also a challenge to interpret the evidence provided in terms of validity and responsiveness as no hypothesis about these aspects of the 
instruments are formulated and tested in such studies;

3. Literature that focuses on instruments that measure women's level of satisfaction with their care, rather than their actual experience of that care, as per the methodological limitations with satisfaction as discussed earlier in this paper;

4. Literature that focuses on care received from a specific profession, e.g. midwives, as opposed to a measurement of women's experiences of their maternity care in general;

5. Literature that focuses on brief versions of full instruments that have been reported elsewhere; and

6. Childbirth experiences that merit specific consideration, for example stillbirths. These experiences require more specific approaches based on the needs and experiences of women in these groups.

\section{Data extraction process}

A prespecified data extraction form will be designed and populated with data extracted from each study. This form will be piloted on three studies independently by two reviewers and compared to ensure consistency in the interpretation of the data being extracted and modified as required. Based on the expectation that some instruments will be the focus of multiple studies, these studies will be grouped together to reduce data extraction duplication [21]. Where the same data has been reported in more than one place, the more comprehensive version will be included.

A stepped approach will be employed to facilitate the extraction of data. Double data extraction will commence with both reviewers independently analysing $10 \%$ of the overall number of studies for inclusion in the review. The results of this initial analysis will be compared, and errors resolved through discussion. If significant discrepancies ( $>10 \%$ of all data items extracted per study) are apparent, then double data extraction will continue for a further $10 \%$ of studies. The results will again be compared, and errors discussed. If there are still significant discrepancies at this stage of the analysis, double data extraction will continue for all studies for inclusion in the review. If significant discrepancies are not apparent at this stage, each reviewer will take $50 \%$ of the remaining studies and extract data independently. Disagreements will be resolved through consensus with a third reviewer.

\section{Evaluation of methodological quality and quality of results}

The COnsensus-based Standards for the selection of health Measurement Instruments (COSMIN) steering committee have developed a guideline for systematic reviews of patient-reported outcome measures (PROM) [22]. This guideline, adapted as necessary to suit this review, will be used to guide the evaluation of the measurement properties of each included study.

Each measurement property will be evaluated via a three-step process: (1) evaluate the methodological quality of each included study, (2) evaluate the criteria for good measurement properties using quality criteria and (3) summarise the evidence.

1. Evaluate the methodological quality of each included study: This COSMIN Risk of Bias checklist $[23,24]$ is an adaptation of the original COSMIN checklist [25]. The Risk of Bias checklist has been developed specifically for use in systematic reviews of PROMs to assess the risk of bias of studies on measurement properties.

The checklist contains one box on development and nine boxes for measurement properties (content validity, structural validity, internal consistency, cross-cultural validity/measurement invariance, reliability, measurement error, criterion validity, hypothesis testing for construct validity and responsiveness) [23]. The COSMIN expert group reached international consensus on the taxonomy, terminology and definitions of these measurement properties; full explanations are available from Mokkink et al. [26].

Each box contains between 3 and 35 items and applicable items will be scored for each included study based on a four-point rating scale (i.e. 'inadequate', 'doubtful', 'adequate' or 'very good'). An overall score for the methodological quality of a given measurement property is determined by the lowest rating that is assigned to the items within a given box [23].

2. Evaluate the criteria for good measurement properties using quality criteria: The measurement properties of each included instrument will be evaluated using the COSMIN recommended criteria for good measurement properties, as outlined in Table 2 [15, 22, 27].

The reported findings for each measurement property will be compared with the predetermined criteria described in Table 2, and the adequacy of results of each study will be rated as sufficient (+), intermediate (?) or insufficient (-).

3. Summarising the evidence: The results retrieved from 1 and 2 above will be evaluated for consistency across studies. If the results are consistent they will be summarised, compared against the criteria for good measurement 
Table 2 COSMIN recommended criteria for good measurement properties

\begin{tabular}{|c|c|c|}
\hline Measurement property & Rating & Criteria \\
\hline \multirow[t]{3}{*}{ Structural validity } & + & $\begin{array}{l}\text { Classical Test Theory (CTT) } \\
\text { Confirmatory factor analysis: Comparative Fit Index or Tucker Lewis Index or comparable measure }>0.95 \\
\text { OR root mean square error of approximation }<0.06 \text { OR standardised root mean residuals }<0.08 \\
\text { Item Response Theory (IRT)/Rasch } \\
\text { No violation of unidimensionality: Comparative Fit Index or Tucker Lewis Index or comparable measure }> \\
0.95 \text { OR root mean square error of approximation }<0.06 \text { OR standardised root mean residuals }<0.08 \\
\text { AND } \\
\text { No violation of local independence: residual correlations among the items after controlling for the } \\
\text { dominant factor }<0.20 \text { OR Q3's }<0.37 \\
\text { AND } \\
\text { No violation of monotonicity: adequate looking graphs OR item scalability }>0.30 \\
\text { AND } \\
\text { Adequate model fit: } \\
\text { IRT: } x 2>0.001 \\
\text { Rasch: infit and outfit mean squares } \geq 0.5 \text { and } \leq 1.5 \text { OR Z-standardized values }>-2 \text { and }<2\end{array}$ \\
\hline & $?$ & $\begin{array}{l}\text { CTT: not all information for ' }+ \text { ' reported } \\
\text { IRT/Rasch: model fit not reported }\end{array}$ \\
\hline & - & Criteria for ' + ' not met \\
\hline \multirow[t]{3}{*}{ Internal consistency } & + & $\begin{array}{l}\text { At least low evidence for sufficient structural validity AND Cronbach's alpha(s) } \geq 0.70 \text { for each } \\
\text { unidimensional scale or subscale }\end{array}$ \\
\hline & $?$ & Criteria for 'At least low evidence for sufficient structural validity' not met \\
\hline & - & $\begin{array}{l}\text { At least low evidence for sufficient structural validity AND Cronbach's alpha(s) }<0.70 \text { for each } \\
\text { unidimensional scale or subscale }\end{array}$ \\
\hline \multirow[t]{3}{*}{ Reliability } & + & Interclass Correlation Coefficient (ICC) or weighted kappa $\geq 0.70$ \\
\hline & $?$ & ICC or weighted kappa not reported \\
\hline & - & ICC or weighted kappa $<0.70$ \\
\hline \multirow[t]{3}{*}{ Measurement error } & + & Smallest detectable change (SDC) or limits of agreement (LoA) < minimal important change (MIC) \\
\hline & $?$ & MIC not defined \\
\hline & - & SDC or LoA > MIC \\
\hline \multirow{3}{*}{$\begin{array}{l}\text { Hypotheses testing for construct } \\
\text { validity }\end{array}$} & + & The result is in accordance with the hypothesis \\
\hline & $?$ & No hypothesis defined (by the review team) \\
\hline & - & The result is not in accordance with the hypothesis \\
\hline \multirow[t]{3}{*}{$\begin{array}{l}\text { Cross-cultural validity/ } \\
\text { measurement invariance }\end{array}$} & + & $\begin{array}{l}\text { No important differences found between group factors (e.g. age and language) in multiple group factor } \\
\text { analysis OR no important differential item functioning (DIF) for group factors (McFadden's } R<0.02 \text { ) }\end{array}$ \\
\hline & $?$ & No multiple group factor analysis OR DIF analysis performed \\
\hline & - & Important differences between group factors OR DIF was found \\
\hline \multirow[t]{3}{*}{ Criterion validity } & + & Correlation with gold standard $\geq 0.70$ OR area under the curve (AUC) $\geq 0.70$ \\
\hline & $?$ & Not all information for ' + ' reported \\
\hline & - & Correlation with gold standard $<0.70$ OR AUC $<0.70$ \\
\hline \multirow[t]{3}{*}{ Responsiveness } & + & The result is in accordance with the hypothesis $O R A \cup C \geq 0.70$ \\
\hline & $?$ & No hypothesis defined (by the review team) \\
\hline & - & The result is not in accordance with the hypothesis OR AUC $<0.70$ \\
\hline
\end{tabular}

properties and deemed as (+) sufficient, (-) insufficient, $( \pm)$ inconsistent or intermediate (?). As per COSMIN guidance [22], we will explore any inconsistency in results. If an explanation is found, an overall rating of the instrument will be provided for several subgroups. The subgroups of interest include ethnicity, religion, sexual orientation, delivery type, age of participants (e.g. under 18), type of country (developing vs. developed), setting (public hospital, private clinic, midwife led, etc.), multiple vs. singleton pregnancy and nulliparous vs. multiparous women.

\section{Additional evaluation}

As per the stated study objectives, additional evaluations will be made on the following:

1. Items included within each survey instrument: 
The purpose of categorising items that are included within each instrument is to evaluate what aspects of care are being measured as it has been acknowledged that a limitation of survey instruments, and possibly the reason why there has been a proliferation of such instruments in recent times, is that feedback is provided only on aspects of care that are included specifically [9]. The inclusion of this objective allows for the identification of the most commonly used items within survey instruments that encompass the entire maternity care process and will highlight gaps or inconsistencies across the domains of all instruments. A data extraction form has been developed to standardise the collection of information related to the items included within each survey instrument and to aid analysis (Table 3). All relevant information that is available within the included studies will be extracted as direct quotes to populate the data extraction form

\section{Planned methods of analysis}

General information and instrument detail will be summarised in tabular format. The reasons for excluding studies at full text will also be reported in tabular format. The analysis of each retrieved item pool and the use of this in later phases of the project will be published separately.

The methodological quality and results of each instrument will be compared using a structured narrative synthesis. Additionally, an overview of the combined results of measurement properties will be summarised in tabular

Table 3 Data extraction form

\begin{tabular}{ll}
\hline General & Original author \\
information & Title \\
& Journal \\
& Year \\
& Country of origin \\
& Language and available translations \\
& Background of the jurisdictions healthcare \\
& Study design \\
& Study aim \\
Instrument details/ & Outcome measure \\
items included & Purpose/use \\
& Number of domains \\
& Number of items \\
& Structure of domains and items \\
& List of complete bank of items included ${ }^{a}$ \\
& Scale design (type of scale, e.g Likert, no. of points \\
& of scale) \\
& Target population - inclusion and exclusion criteria
\end{tabular}

${ }^{\mathrm{a}}$ Bank of items included within each survey instrument to be recorded within an additional file under the headings antenatal, intranatal, postnatal and misc format as described above under the subheading 'Summarising the evidence'. This combination of analysis will allow for recommendations to be made on the use, adaptation and development of future survey instruments measuring women's experiences of their maternity care.

\section{Discussion}

The value of survey instruments that measure women's experiences of their maternity care has been recognised internationally with many countries employing the use of such instruments to inform policy and practice. In influencing policy and practice, the data arising from these instruments is used to direct limited resources within maternity services. As such, the development of instruments must be methodologically sound and the instrument itself fit for the purpose and context in which it is used to ensure the resulting data is credible. This systematic review will serve as a guide for choosing the most appropriate existing instruments to use or adapt so that they are fit for purpose, in addition to informing the development of new instruments. This review is timely, not only as it fills a gap in the current literature, but also because the use and development of such instruments is increasing internationally.

\section{Abbreviations}

AUC: Area under the curve; CINAHL: Cumulative Index of Nursing and Allied Health Literature; COSMIN: COnsensus-based Standards for the selection of health Measurement Instruments; CTT: Classical Test Theory; DIF: Differential item functioning; EMBASE: Excerpta Medica dataBASE; ICC: Interclass

correlation coefficient; IRT: Item Response Theory; LoA: Limits of agreement; MEDLINE: MEDLINE is the online counterpart to MEDLARS MEDical Literature Analysis and Retrieval System; MIC: Minimal important change; PRISMAP: Preferred Reporting Items for Systematic Reviews and Meta-Analyses- Protocols; PRISMA: Preferred Reporting Items for Systematic Reviews and MetaAnalyses; PROM: Patient-reported outcome measure; PROSPERO: International Prospective Register of Systematic Reviews; SDC: Smallest detectable change

\section{Acknowledgements}

Not applicable.

Authors' contributions

I, $C B$ as guarantor of the review, confirm that all authors $(C B, R G, L O$, $D, E R$, $\mathrm{MW}, \mathrm{MB}$ and $\mathrm{DD}$ ) meet the criteria for authorship and have approved the final article and that all those entitled to authorship are listed as authors.

\section{Funding}

Claire Beecher (corresponding author) is being funded by the Programme for Health Service Improvement, Health Service Executive. Dr. Mark White, Programme Integration Manager, of this programme has contributed as a co- author on this manuscript. No other contribution by this funding body was made to this manuscript.

Availability of data and materials

Not applicable.

Ethics approval and consent to participate

Not applicable.

Consent for publication

Not applicable

Competing interests

The authors declare that they have no competing interests. 


\section{Author details}

'School of Nursing and Midwifery, National University of Ireland, Galway, Ireland. ${ }^{2}$ National Perinatal Epidemiology Centre, Department of Obstetrics and Gynaecology, Cork University Maternity Hospital, Cork, Ireland. ${ }^{3}$ Department of Measurement, Evaluation, Statistics \& Assessment, Boston College, Chestnut Hill, Massachusetts, USA. ${ }^{4}$ Department of Paediatrics, University Hospital Galway, Galway, Ireland. ${ }^{5}$ Programme for Health Service Improvement, Health Service Executive, Dublin, Ireland. ${ }^{6}$ Department of Nursing, University of the Highlands and Islands, Inverness, Scotland. 'Health Research Board - Trials Methodology Research Network (HRB-TMRN), Galway, Ireland. ${ }^{8}$ Evidence Synthesis Ireland, Galway, Ireland.

Received: 17 August 2018 Accepted: 20 December 2019 Published online: 06 January 2020

\section{References}

1. Sixma HJ, Kerssens JJ, Campen CV, Peters L. Quality of care from the patients' perspective: from theoretical concept to a new measuring instrument. Health Expect. 1998;1(2):82-95.

2. Scales CD Jr, Schulman KA. Triggering management for quality improvement. Health Serv Res. 2014;49(5):1401-6.

3. Abdellah FG, Levine E. Developing a measure of patient and personnel satisfaction with nursing care. Nurs Res. 1957;5(3):100-8.

4. Wiegers TA. The quality of maternity care services as experienced by women in the Netherlands. BMC Pregnancy Childbirth. 2009;9:18.

5. Crow R, Gage H, Hampson S, Hart J, Kimber A, Storey L, et al. The measurement of satisfaction with healthcare: implications for practice from a systematic review of the literature. Health Technol Assess (Winchester, England). 2002;6(32):1-244

6. Fitzpatrick R. Surveys of patients satisfaction: I--Important general considerations. BMJ. 1991;302(6781):887-9.

7. van Teijlingen ER, Hundley V, Rennie AM, Graham W, Fitzmaurice A. Maternity satisfaction studies and their limitations: "what is, must still be best". Birth (Berkeley, Calif). 2003;30(2):75-82

8. Perriman N, Davis D. Measuring maternal satisfaction with maternity care: a systematic integrative review. What is the most appropriate, reliable and valid tool that can be used to measure maternal satisfaction with continuity of maternity care? Women Birth. 2016;29(3):293-9.

9. Graham C, Woods P. Patient Experience Surveys. In: Ziebland S, Coulter A, Calabrese JD, Locock L, editors. Understanding and using health experiences improving patient care. Oxford: Oxford University Press; 2013. p. 81-93.

10. Bureau of Health Information. Patient Perspectives - Experiences of maternity care in NSW public hospitals, January to December 2015. Sydney: Bureau of Health Information; 2017.

11. Declercq E, Chalmers B. Mothers' reports of their maternity experiences in the USA and Canada. J Reprod Infant Psychol. 2008:26(4):295-308.

12. Care Quality Commission. Maternity services survey 2015: Care Quality Commission; 2015. Available from: http://www.cqc.org.uk/content/ maternity-services-survey-2015.

13. Scheerhagen M, van Stel HF, Birnie E, Franx A, Bonsel GJ. Measuring client experiences in maternity care under change: development of a questionnaire based on the WHO Responsiveness model. PLoS One. 2015; 10(2):e0117031.

14. Mokkink LB, Terwee CB, Stratford PW, Alonso J, Patrick DL, Riphagen I, et al. Evaluation of the methodological quality of systematic reviews of health status measurement instruments. Qual Life Res. 2009;18(3):313-33.

15. Terwee CB, Bot SD, de Boer MR, van der Windt DA, Knol DL, Dekker J, et al. Quality criteria were proposed for measurement properties of health status questionnaires. J Clin Epidemiol. 2007:60(1):34-42.

16. Moher D, Shamseer L, Clarke M, Ghersi D, Liberati A, Petticrew M, et al. Preferred reporting items for systematic review and meta-analysis protocols (PRISMA-P) 2015 statement. Syst Rev. 2015;4:1.

17. Moher D, Liberati A, Tetzlaff J, Altman DG. Preferred reporting items for systematic reviews and meta-analyses: the PRISMA statement. J Clin Epidemiol. 2009;62(10):1006-12.

18. Messent P. Professional issues. Evaluating women's experiences for a MSLC.. Maternity Services Liaison Committee. British Journal of Midwifery. 2002; 10(10):626-30.

19. Covidence systematic review software. Covidence systematic review software Melbourne, Australia: Veritas Health Innovation; 2018. Available from: https://www.covidence.org/home.
20. Terwee $\mathrm{C}$, de Vet H, Prinsen C, Mokkink L. Protocol for systematic reviews of measurement properties. COSMIN: COSMIN; 2011. Available from: http:// www.cosmin.nl/images/upload/files/Protocol\%20klinimetrische\%20review\%2 Oversion\%20nov\%202011.pdf.

21. Beattie M, Murphy DJ, Atherton I, Lauder W. Instruments to measure patient experience of healthcare quality in hospitals: a systematic review. Syst Rev. 2015;4:97.

22. Prinsen CAC, Mokkink LB, Bouter LM, Alonso J, Patrick DL, de Vet HCW, et al. COSMIN guideline for systematic reviews of patient-reported outcome measures. Qual Life Res. 2018;27(5):1147-57.

23. Mokkink LB, de Vet HCW, Prinsen CAC, Patrick DL, Alonso J, Bouter LM, et al. COSMIN Risk of Bias checklist for systematic reviews of patient-reported outcome measures. Qual Life Res. 2018:27(5):1171-9.

24. Terwee CB, Prinsen CAC, Chiarotto A, Westerman MJ, Patrick DL, Alonso J, et al. COSMIN methodology for evaluating the content validity of patient-reported outcome measures: a Delphi study. Qual Life Res. 2018; 27(5):1159-70.

25. Mokkink LB, Terwee CB, Patrick DL, Alonso J, Stratford PW, Knol DL, et al. The COSMIN checklist for assessing the methodological quality of studies on measurement properties of health status measurement instruments: an international Delphi study. Qual Life Res. 2010;19(4):539-49.

26. Mokkink LB, Terwee CB, Patrick DL, Alonso J, Stratford PW, Knol DL, et al. The COSMIN study reached international consensus on taxonomy, terminology, and definitions of measurement properties for health-related patient-reported outcomes. J Clin Epidemiol. 2010;63(7):737-45.

27. Prinsen CA, Vohra S, Rose MR, Boers M, Tugwell P, Clarke M, et al. How to select outcome measurement instruments for outcomes included in a "core outcome set" - a practical guideline. Trials. 2016;17(1):449.

\section{Publisher's Note}

Springer Nature remains neutral with regard to jurisdictional claims in published maps and institutional affiliations.

Ready to submit your research? Choose BMC and benefit from:

- fast, convenient online submission

- thorough peer review by experienced researchers in your field

- rapid publication on acceptance

- support for research data, including large and complex data types

- gold Open Access which fosters wider collaboration and increased citations

- maximum visibility for your research: over $100 \mathrm{M}$ website views per year

At $\mathrm{BMC}$, research is always in progress.

Learn more biomedcentral.com/submission 